\title{
Towards a Context-Sensitive System To Support Crisis Communication Message Dissemination
}

\author{
Thiago Rodrigues \\ Federal University of Bahia \\ Department of Computer \\ Science \\ Salvador, BA, Brazil \\ thiagonr86@gmail.com
}

\author{
Felipe A. L. Machado \\ Federal University of Bahia \\ Department of Computer \\ Science \\ Salvador, BA, Brazil \\ alm.felipe@gmail.com
}

\author{
Jaziel Souza Lobo \\ Federal Institute of Sergipe \\ Federal University of Bahia \\ Department of Computer \\ Science \\ Salvador, BA, Brazil \\ jaziel.lobo@gmail.com
}

\author{
Vaninha Vieira \\ Federal University of Bahia \\ Department of Computer \\ Science \\ Salvador, BA, Brazil \\ vaninha@dcc.ufba.br
}

\author{
Manoel C. M. Neto \\ Federal Institute of Bahia \\ Department of Computer \\ Science \\ Salvador, BA, Brazil \\ manoelnetom@gmail.com
}

\begin{abstract}
Identifying potential victims to avoid alert people who are not in danger is an open challenge to crisis communications systems. In an emergency situation alerting individuals out of danger can overload dissemination systems and lead to loss of system credibility because people can be overwhelmed with irrelevant information. This paper aims to investigate how to improve the message dissemination process in crisis communication by using context-aware computing concepts. We propose a system to identify potential victims and define a strategy with a better way to contact them. For evaluation we performed two case studies with real emergency messages. Finally, we discuss ways to identify potential victims of emergency situations.
\end{abstract}

\section{CCS Concepts}

-Human-centered computing $\rightarrow$ Ubiquitous and mobile computing systems and tools;

\section{Keywords}

Context-awareness; Context-Sensitive Systems; Mobile Computing; Crisis Communication; Emergency Management.

\section{INTRODUCTION}

Most emergency situation outcomes are devastating. Every year more than 195 million people around the world are victims of floods [17]. In Brazil, more than $40.9 \%$ of towns were affected by disaster situations between 2008 and 2013 .

Permission to make digital or hard copies of all or part of this work for personal or classroom use is granted without fee provided that copies are not made or distributed for profit or commercial advantage and that copies bear this notice and the full citation on the first page. To copy otherwise, or republish, to post on servers or to redistribute to lists, requires prior specific permission and/or a fee.

SBSI 2017 June $5^{\text {th }}-8^{\text {th }}, 2017$, Lavras, Minas Gerais, Brazil

Copyright SBC 2017.
However, only $4.4 \%$ of Brazilian towns have an early warning system (EWS) [7].

An emergency is a sudden event that calls for immediate measures to minimize its adverse consequences [5]. An emergency can also be defined as a situation which threatens serious damage to human welfare, to the environment, or security of a country [14]. It can be caused by natural phenomena (e.g., floods, tsunami) or by man (e.g., fire, gas leaks) [5].

In an emergency, there are many kind of actors such as operational forces, population, press, volunteers and so on. Operational forces are the firefighters, the police, the command center and any other player trained to support the population. Emergency situations require efficient ways of communication among operational forces and the population at risk. Communication is a key factor to reduce the damage caused by the incident [12]. This kind of communication is known as crisis communication.

Some of the requirements and challenges of crisis communication include: (i) the use of communication systems is not the central activity of people in these situations; (ii) useful instructions should be provided with clear, non ambiguous, and easy to understand information; (iii) a person's context (e.g., spoken language, age, impairment) can influence the message understanding, thus should be used to personalize messages; and (iv) potential victims should be identified and contacted as fast as possible [16, 8]. Context-aware computing provide technologies that support dealing with these challenges [8]. A context-aware system is one that uses relevant information from the environment based on the activity that the user is performing [15]. Context information can be user profiles, environmental data (e.g. temperature, weather), network status, or any other relevant information.

One of the most challenging issues in crisis communication systems is to identify potential victims in the most adequate way to avoid contacting people who are not in danger. This can generate panic among people. Besides this, it can overload dissemination systems as a large number of users can be notified. Also, alerting people who are not in danger can lead to loss of credibility because users can be overwhelmed 
with irrelevant information.

Another challenging in crisis communication systems is to decide how to reach the people at risk using the more appropriate content, communication channels and media formats. The most of the crisis communication solutions not concerns in creating a communication strategy based on contextual information. Therefore, these solutions, normally, does not use approaches to choose the appropriate way to communicate the users for each situation and context.

To support overcoming this problem, in this paper we propose a conceptual architecture for building context-aware crisis communication systems and a context model to represent relevant contextual information in this scenario. To evaluate this architecture, a prototype system was implemented. Context is used to support identifying potential victims, personalize message content, choose message format, and choose the communication channel. To evaluate the prototype system and our context model, we performed two case studies using real emergency data. Finally, we discuss different ways to identify potential victims and how to reach them.

This paper is organized as follows: Section 2 provides background concepts and section 3 discuss related works. Section 4 presents our proposal of the conceptual architecture to aid message dissemination for crisis communication and the conceptual context model. Section 5 presents the implementation of a system prototype. The evaluation is discussed in Section 6 and Section 7 presents the conclusions.

\section{BACKGROUND}

Context has several definitions. However, the most cited is Dey, Abowd and Salber [4] that says: "Context is any information that can be used to characterize the situation of entities (i.e., whether a person, place, or object) that are considered relevant to the interaction between an user and an application, including the user and the application themselves. Context is typically the location, identity, and state of people, groups, and computational and physical objects".

Vieira et al. [18], extends Dey's definition introducing the concept of contextual element (CE). CE is any piece of data that can be used to characterize an entity in an application domain. For example, in the domain of crisis communication, the emergency is an entity which has many contextual elements such as severity, danger zone, and others.

CAP (Common Alerting Protocol) is a standard worldwide used and well-referenced by the scientific community [3] that contains several contextual elements about emergencies. It supports exchanging disaster alerts and public warnings (messages) over all kinds of networks. CAP is an open standard, non-proprietary and XML-based [13]. Code 1 exemplifies the structure of a CAP file with some important fields such as: (i) identifier; (ii) sender - the creator of CAP; (iii) sent - date of CAP creation and dissemination; (iv) severity; (v) headline - short information about the disaster; (vi) description - Detailed information about the disaster; (vii) polygon - geographic coordinates that represents the danger zone of an emergency situation; and (viii) resource - reference to further multimedia information (e.g. images, audios, videos) having its kind, description, and link provided by the CAP sender;

In order to build context-sensitive systems is needed represent the contextual elements and behaviors through a con-

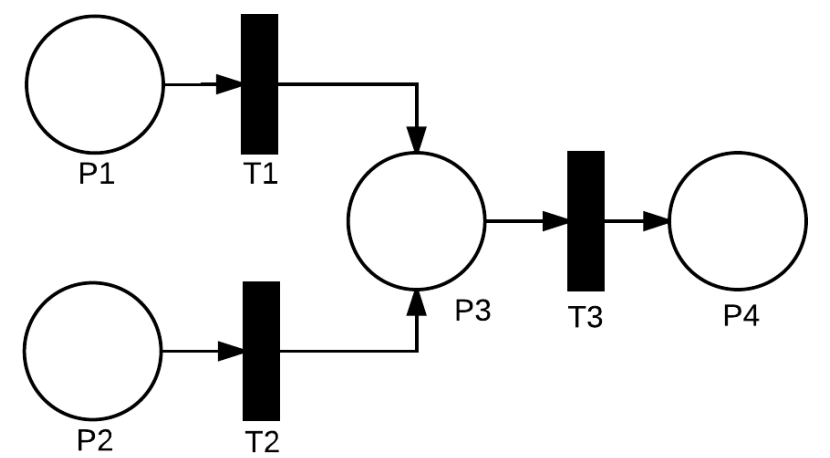

Figure 1: Petri Net example

text model. Some examples of representation techniques are key-value pair, UML, ontology, Petri Net (PN) and so on. $\mathrm{PN}$ is a mathematical modeling language that has a graphical notation and a formal definition for its execution semantics [11]. In this way, PN has the advantages of graphical models (i.e. ease concepts design, specification, and defining context requirements [15]) and advantages of logic based models (i.e. express policies, rules, constraints, and preferences [1]). PN graphical representation is a directed graph whose nodes are depicted as Transitions (T) or Places (P) as shown in the Figure 1. Transitions are actions represented by bars and Places are states represented by circles. Graph directed arcs (represented by arrows) connects a $\mathrm{P}$ to a $\mathrm{T}$ or a $\mathrm{T}$ to a $\mathrm{P}$.

\section{RELATED WORKS}

Our work makes special use of the headline, description, polygon and resources fields of CAP. The polygon field is used to find users at risk whereas headline, description, and resource are used to inform the users.

There are some studies that use context-sensitive approach to support crisis communication. This section presents and compares with our proposal some of these solutions. For this comparison, we examined whether the studies: (i) identify the potential victims; (ii) adapt the message content based on user context; and (iii) enable a choice of communication channels and media format also based on context information. Table 1 shows the comparative analysis of the presented related works and our proposal.

CAP-ONES [10] is an ontology-based system for managing alert notifications. It uses emergency and user context information to personalize the message content and format (focus on disabled people). It chooses the communication channel (e.g., SMS, e-mail) using user preferences. CAP-ONES does not identify potential victims rather, it disseminates alerts to all the subscribed users.

Klafft and Ziegler [9] propose a system prototype that uses multiple communication channels and personalized messages. It provides message content personalization for disabled people, families with children, senior citizens, and tourists (translated content). Their system chooses the message format according to the communication channel. Klafft and Ziegler system prototype always notify in broadcast using all available communication channels. It does not offer a choice of communication channels based on user context and does not identify potential victims.

Global Disaster Alert and Coordination System (GDACS) 


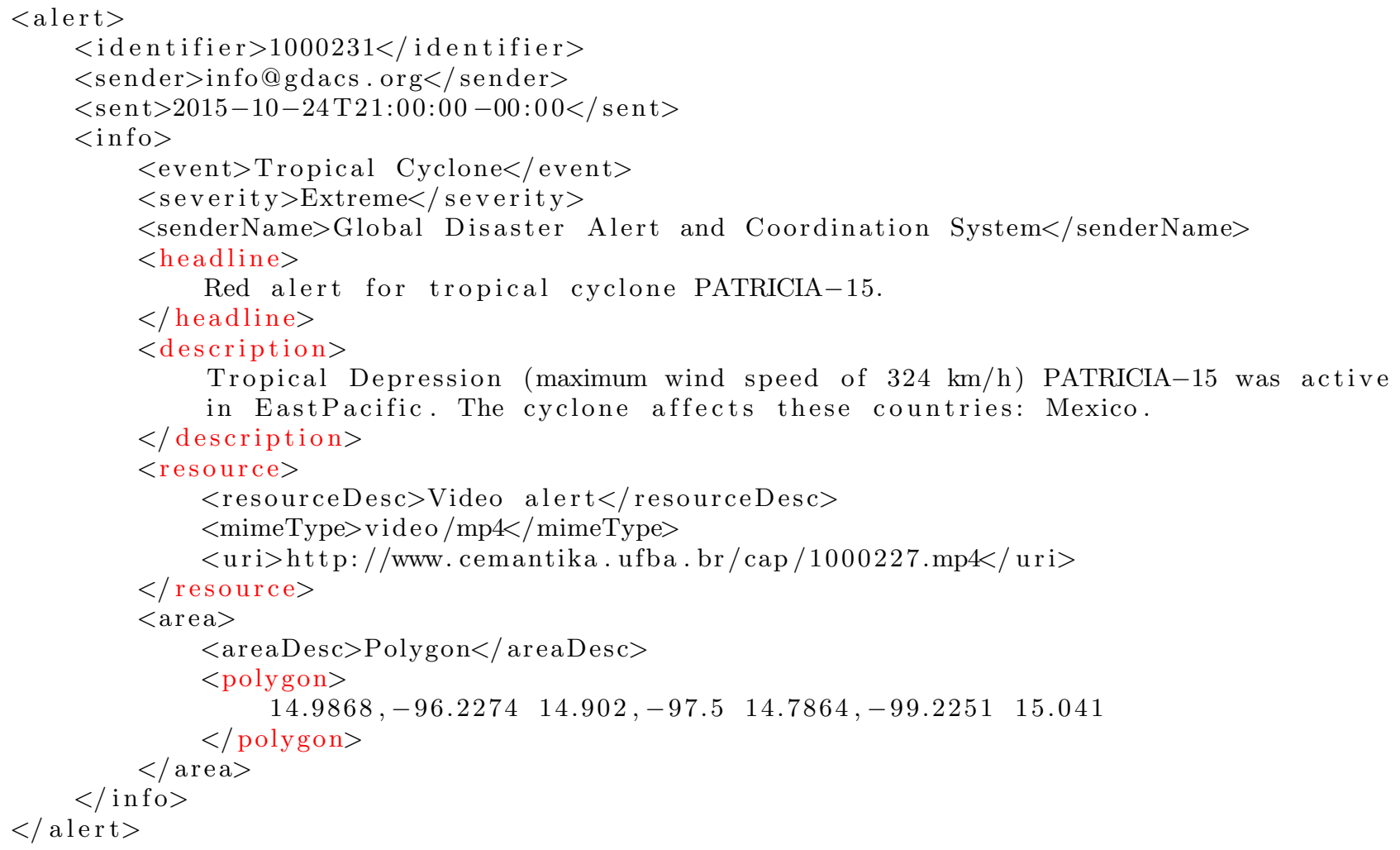

Code 1: CAP file Sample

Table 1: Related Work

\begin{tabular}{|l|l|l|l|l|}
\hline & $\begin{array}{l}\text { Personalize } \\
\text { Message } \\
\text { Content }\end{array}$ & $\begin{array}{l}\text { Adaptive } \\
\text { Message } \\
\text { Format } \\
\text { Selection }\end{array}$ & $\begin{array}{c}\text { Adaptive } \\
\text { Comm. } \\
\text { Channel } \\
\text { Selection }\end{array}$ & $\begin{array}{l}\text { Identify } \\
\text { Potential } \\
\text { Victims }\end{array}$ \\
\hline $\begin{array}{l}\text { Malizia } \\
\text { et al. } \\
{[10]}\end{array}$ & Yes & Yes & Yes & $\begin{array}{l}\text { No, all } \\
\text { sub- } \\
\text { scribers. }\end{array}$ \\
\hline $\begin{array}{l}\text { Klafft } \\
\text { and } \\
\begin{array}{l}\text { Ziegler } \\
{[9]}\end{array}\end{array}$ & Yes & Yes & No & $\begin{array}{l}\text { No, } \\
\text { broad- } \\
\text { cast. }\end{array}$ \\
\hline $\begin{array}{l}\text { GDACS } \\
{[6]}\end{array}$ & No & No & Yes & $\begin{array}{l}\text { Yes, all } \\
\text { users on } \\
\text { conti- } \\
\text { nent. }\end{array}$ \\
\hline $\begin{array}{l}\text { Banerjee } \\
\text { et al. } \\
{[2]}\end{array}$ & Yes & No & No & $\begin{array}{l}\text { Yes, zip } \\
\text { code ap- } \\
\text { proach. }\end{array}$ \\
\hline $\begin{array}{l}\text { Our } \\
\text { Work }\end{array}$ & Yes & Yes & Yes & $\begin{array}{l}\text { Yes, } \\
\text { CAP } \\
\text { Polygon }\end{array}$ \\
\hline
\end{tabular}

[6] is a cooperation between the United Nations, the European Commission and disaster managers worldwide. GDACS has a wide database about previous emergency situations and provides a notification service regarding emergency situations in real time. GDACS does not personalize content messages. Users can choose SMS or e-mail as the commu- nication channel. GDACS consider all user on a continent where an emergency occurs as potential victim and notifies them all.

Banerjee et al. [2] proposed a rule-based solution to deliver alerts to targeted users. Their solution translates message content to the user in their preferred language. However, it does not provide any way to personalize message content nor change the communication channel. The rulebased approach is used to identify potential victims comparing user and emergency zip code. The problem with this method is that it is difficult to scale up to a worldwide emergency notification system.

Related work analysis shows that [10] and [9] broadcast all emergency messages regardless whether users are potential victims or not. GDACS [6] uses a large area (all a continent) as a potential victim delimiter. These three works generally notify many more people than the potential victims. Banerjee et al. [2] introduce an interesting approach for small areas such as a city, but it is hard to scale it to a worldwide system.

\section{A CONTEXT-SENSITIVE SYSTEM TO SUPPORT CRISIS COMMUNICATION}

This section presents our proposal for a context-sensitive system to support dissemination of crisis communication messages. First we describe the system conceptual architecture, then we present its context model.

\subsection{Conceptual Architecture}

To support message dissemination for crisis communica- 
tion systems, we defined a conceptual architecture as depicted in Figure 2. The architecture consists of three main components: Context Manager, Message Builder and Disseminator.

The Context Manager component handles the acquisition, interpretation, and reasoning of contextual information. The context information can be acquired from different sources, such as people, devices such as smartphones and other systems. The Context Manager receives the information, process according the domain necessity and applies reasoning rules in order to obtain relevant information for the next layers of architecture.

To process contextual information the proposed solution needs to implement a context model that defines the entities, contextual elements, and their behavior. A conceptual context model to support our architecture is presented in the next section.

The Message Builder component identifies the individuals who need to be notified about an emergency. For this, the Message Builder must use information from the Context Manager component. As well as, this component performs necessary adaptation to the content of messages and defines the strategy for dissemination over different channels and users, and using different media formats (e.g. text, image, audio, video).

The Message Builder output is the input for the Message Disseminator component. Following that, the Disseminator sends the alert messages to receivers through each selected communication channel using the chosen (and available) data format for the content of a message.

\subsection{Conceptual Context Model}

To design context-aware application, identification of entities and contextual elements are needed [18]. For the purpose of building a context-sensitive system to assist message dissemination in emergency situations, three contextual entities were identified: user, emergency, and smartphone.

A user can be a potential victim if $\mathrm{s} /$ he is near an emergency situation. Thus, their location is important as well as the need to contact them as directly as possible. For emergency systems, the location of the user and the emergency is necessary and important to identify potential victims, whereas knowing the user language is also important to translate the information and establish more efficient contact.

This work focuses on the smartphone as a communication device. It is a contextual entity and the contextual elements used to improve communication are connectivity and connection type. Connectivity represents whether the device is connected to the Internet or not. If the device is connected, connection type represents the kind of network connection, such as $\mathrm{Wi}-\mathrm{Fi}, 4 \mathrm{G} / 3 \mathrm{G}$ or $2 \mathrm{G}$.

All context information was used to define rules to support message dissemination for crisis communication systems. These rules, information flow, and process flow are represented in a context model using Petri Net. Our context model solution is shown in Figure 3.

The model depicted in Figure 3 can be divided into two phases. The first one is data acquisition and personalization. The second one is message format and distribution.

The first place in the model is "Emergency data received". This place represents the acquisition of context information about the emergency. Next, transition T1 reads this infor- mation reaching "Emergency data read" place. The transition "Discard user data not in danger" blocks the flow until the system reaches "User data read" state. This transition uses emergency and user data to know which users are in the danger zone. After this, the system reaches the place "Emergency data and user data obtained". Here, the system encounters a list of users in the danger zone, their profile data, and information about the emergency. Transition T4 translates emergency data to the user's preferred language, and then the system reaches on "Translated emergency data and user data obtained" place. Next, transition T5 searches smartphone data from users in danger reaching the next place with all data gathered. This represents the end of the first phase.

The second phase starts when the transition T4 verifies if the user's smartphone have network access. If it is offline, the system arrives the "doesn't have network access" place and the after the transition T8 reaches two places ("SMS selected" and "E-mail selected") at the same time. Then, it actives T10 and T11 parallel and finally the system reaches the place "Message prepared to be send". So, if the user's smartphone is offline, the system chooses to notify the user by SMS (using text) and E-mail (using text and image). Otherwise, if the smartphone is online, the system reaches the place "has network access". Next, T7 selects the smartphone app as communication channel and T9 checks the smartphone connectivity type and the system goes to the respective place (Wi-Fi selected, $4 \mathrm{G}$ or $3 \mathrm{G}$ selected, or $2 \mathrm{G}$ selected). From each one of these, the respective transaction (T12, T13 or T14) are active choosing the message format and, finally, the system reaches the place "Message prepared to be send".

The place "Message prepared to be send" represents the output of the system. It contains a set of users who will be contacted (potential victims), the communication channel that will be used (for each user), the message format (for each communication channel), and the message content in the user's preferred language if it is text.

\section{IMPLEMENTATION}

Based on defined conceptual architecture and context model, we implemented a prototype of a system to support message dissemination for crisis communication. Our prototype is a console application executed by a terminal with parametrized input (CAP emergency files, user profiles, and smartphones data file). The prototype execution follows the Petri Net contextual model sequence, implementing: (i) acquisition of contextual information from the data source files; (ii) content adaptation based on user's and emergency's contexts; and (iii) selection of communication channels and message media formats.

\subsection{Data Acquisition and Adaptation}

The system implements the acquisition of contextual information from different data sources: (i) user data as user profiles; (ii) user's smartphone data; and (iii) emergency information message in CAP format. These files are read from directories set up before the system starts. The input data is used to define which user is a potential victim and needs to be informed about an emergency situation, to personalize message content, and to choose communication channels and message media formats.

The system uses three kinds of information about the user: 


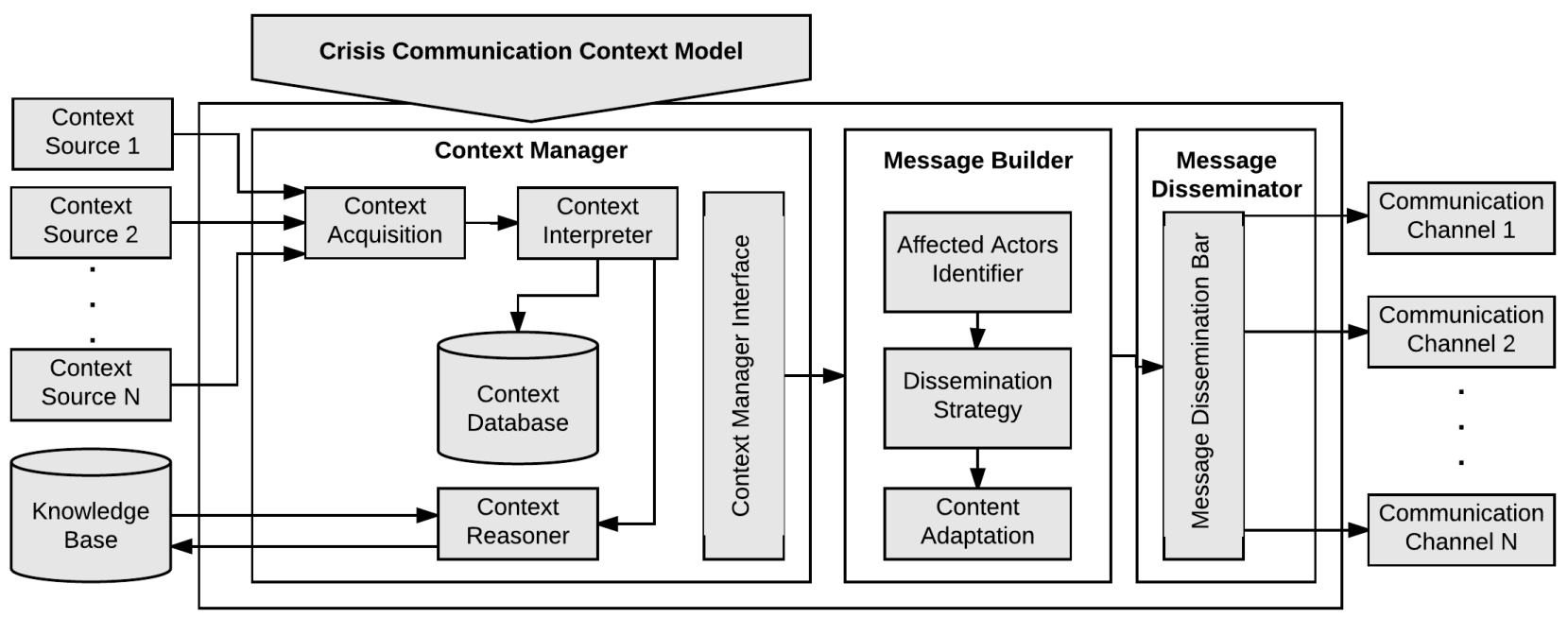

Figure 2: Conceptual Architecture with three main components: Context Manager, Message Builder, and Message Disseminator
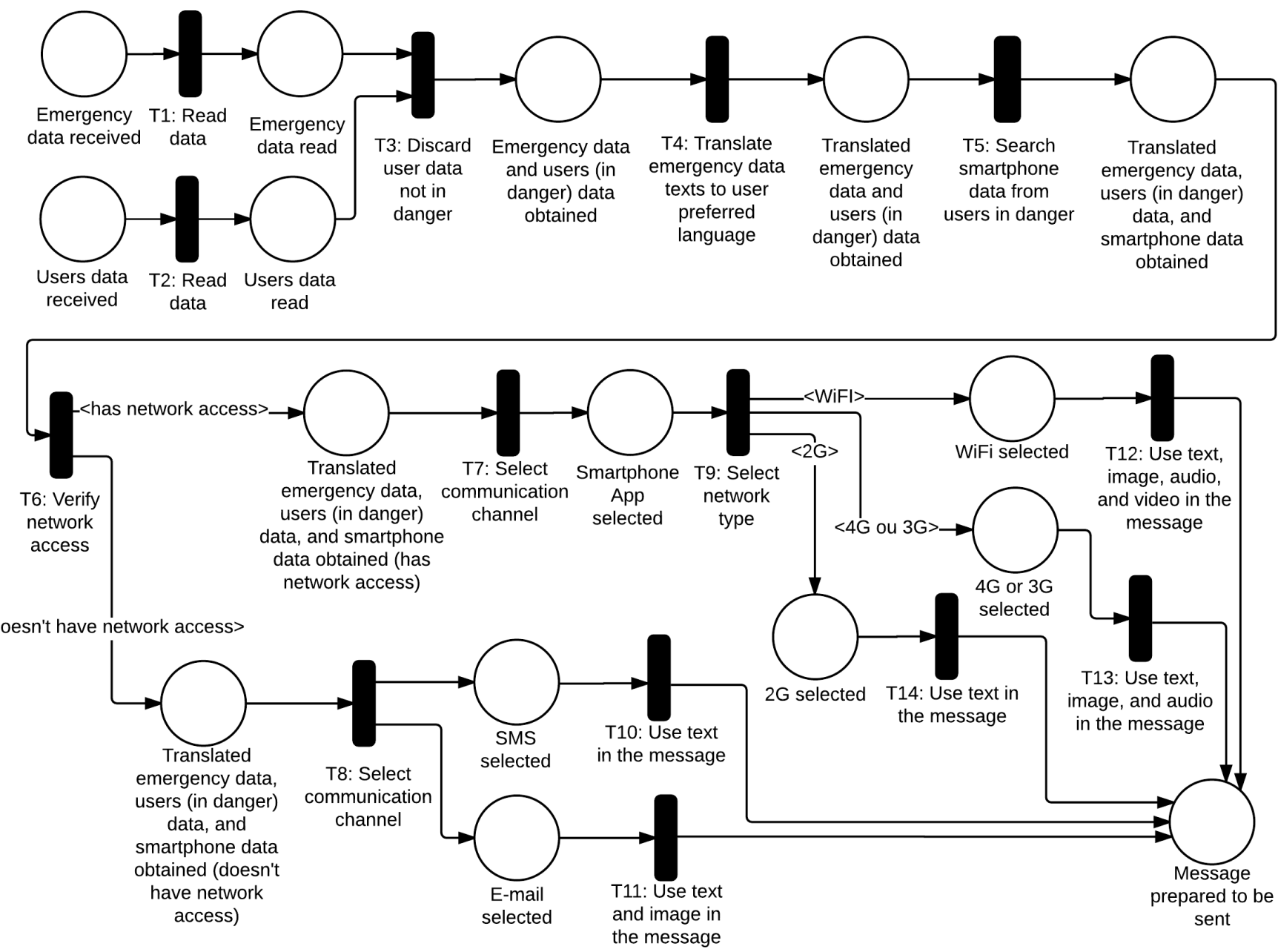

Figure 3: Conceptual Context Model Using Petri Net 
(i) basic information; (ii) communication data; and (iii) location. Basic information about user is identification (id), name, year of birth and preferred language. Communication data are phone number, e-mail, and smartphone operational system (Android or iOS). Location data are the most visited places, such as home and work locations. This information is read from stored user profile files.

Another data source for the system is the user's smartphone. Information about a user's smartphones is important to help to decide whether the user needs to be contacted and how to contact him. Thus, the main information read by the system about the user's smartphone is the last location and the date and time of last location update. As with user's profile, the user's smartphone information also is read from stored files.

CAP message is an important input for the system. A CAP message has a considerable amount of information about a disaster situation. The information extracted from these messages and used by system are: (i) identifier; (ii) Sender; (iii) MsgType - Code denoting the nature of the alert message; (iv) Headline - A brief human-readable headline of the hazard; (v) Description - An description of the hazard that occasioned the message; (vi) Polygon - A geographic polygon of the danger zone represented for $\mathrm{N}$ points consisting of the longitude and latitude that forms an area; and (vii) Resource - Additional file with supplementary information such as images, audios and videos.

Acquisition of emergency data from CAP message files is the first system step. Next, which users are in the danger zone is verified according to users' location and polygon field in the CAP message. To obtain the users' location, the system reads the user profile (location of work and home) and the last smartphone location. A user is in danger if one of their locations is inside the danger zone polygon (from CAP message). To identify these users, we use a Java API that uses the $\mathrm{N}$ points of a CAP polygon to create a geometric form with $\mathrm{N}$ sides and verify if within the area of this polygon there are some of the user locations. A visual representation of this polygon is shown on Figure 4 by the red circle.

Once the list of users in danger has been established, the system prototype performs the content adaptation step. In this case, emergency information is translated into the user's preferred language (from users profile). This step is represented in our context Model as transition T3 (translate emergency data) of Petri Net as depicted in Figure 3. Then, the system generates the final personalized data set.

\subsection{Channel and Message Format Selection}

Selection of the communication channel and message format starts in the transition T4 of our Petri Net. To implement these two steps we adopted a rules-based approach using Drools ${ }^{1}$ tool. Drools is a business rules management system solution that provides an inference machine and its own language for rule descriptions.

We use Drools inference machine to process the two most complex logics in the system. The first one is the choice of communication channel. Here we have three possibilities: smartphone app, SMS and e-mail. The second one is the choice of message media formats based on the user, selected communication channel, and available resources in the CAP message. we defined a strategy for message dissemination,

\footnotetext{
${ }^{1}$ http://www.drools.org/
}

once the system generates an output with the list of users who need be notified, the communication channels to reach these users, and the type of media for each communication channel. The logic of these rules was explained in the Conceptual Context Model section.

\subsection{System Output}

Following all the processing, the system needs to build the output with the dissemination strategy. The system output is an XML file that contains the users who need to be contacted, how to contact them, and the message contents. As shown in the Code 2, the output contains a reference to the CAP message, the number of users at risk, and the list of users with the selected communication channels and the media formats for each channel.

The message content about the emergency included in the file output comes from the CAP as mentioned before. The textual content is extracted from the Headline and Description fields from the CAP and the multimedia content is extracted from the Resource field. An extension to our prototype based on Disseminator layer of conceptual architecture can implement the message dissemination process through this output.

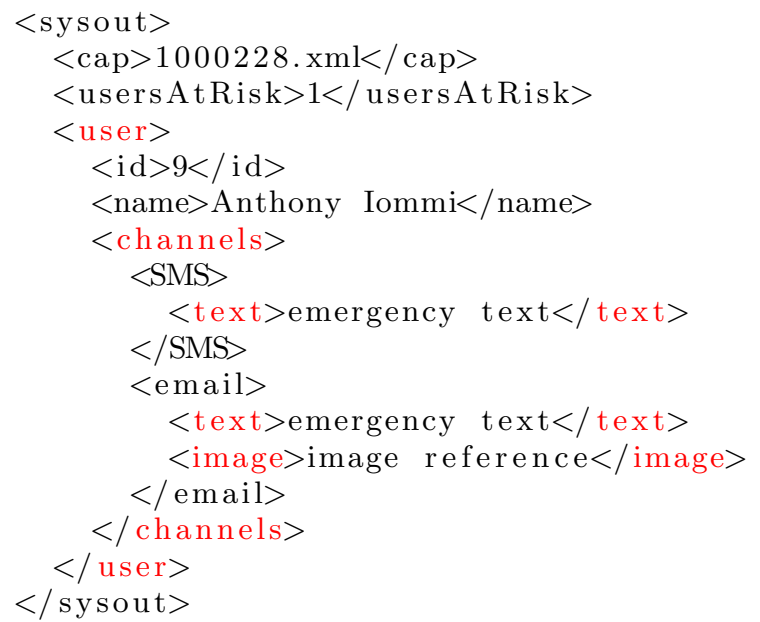

Code 2: Formatted dissemination strategy - For each emergency the users who need to be notified and the channels for this purpose are listed

\section{EVALUATION}

To evaluate our proposal, we perform tests running the system prototype with real emergency messages split into two case studies. The first one by using emergency data from GDACS databases and the second one with emergency data ceded by INMET ${ }^{2}$ (Brazilian National Institute of Meteorology). Emergency data from both sources were formatted as CAP message.

\subsection{GDACS Emergency Data Case Study}

The first case study was performed with GDACS emergency data. To extract these data from GDACS, we developed and used an Internet bot which systematically browses the World Wide Web (Web crawler). The Web crawler downloaded 162 CAP messages. All messages from 2015.

\footnotetext{
${ }^{2}$ http://www.inmet.gov.br
} 
However, 35 messages had no danger zone information and 32 concerned only unpopulated areas (ocean). Thus, we remove these messages. In the end, 95 messages were used in this step of the evaluation process.

All the information about the users was simulated. User profiles were created based on the emergency data. For each emergency situation, we created one user and his geographic location is an arbitrary point inside the danger zone described in the CAP message. We considered that each user had one smartphone and the information about these devices was simulated as well. Information about user smartphones was randomly generated so that they could be up-to-dated with information (user online) and outdated (user offline) information. The user connectivity mode was generated randomly too.

Our first case study was run with 95 emergency scenarios. Each test scenario had at least one user within the danger zone so that one person would need to be contacted in all situations.

\subsection{INMET Emergency Data Case Study}

For the second case study we used a larger number of real emergency data. INMET kindly provided 1321 CAP messages. All the messages were dated between June and December 2015 and cover only situations in Brazil. No message was discarded.

As in the first case study, all the information about the users and the smartphones data were simulated. Each CAP message meant one emergency scenario and each scenario had at least one user within the danger zone. Therefore, 1321 scenarios were performed by the system prototype based on INMET's dataset. The results are discussed below.

\subsection{Results and Analysis}

For both case studies, after the scenarios execution, the system generated the formatted dissemination strategy file as shown in the Code 2. The output indicates which users need to be contacted based on the processing of contextual element (location) for both emergency and user. Contacting only users within the danger zone is the first objective of the system prototype.

Figure 4 is a visual representation of the prototype behavior. The circle is a danger zone. This data is extracted from polygon field of CAP emergency message. The markers are users at their geographic location. This information was acquired using logic described in section 5.1. Only users inside the circles were selected to be contacted.

When a crisis communication system notifies people who are not in danger, these people are considered false positives. Our proposed technique to identify potential victims provides an efficient way to reduce the number of these false positives by using users location and information about the disaster danger area from CAP files. The number of false positives will increase if we apply the approach of notifying the entire country or continent like some other solutions. This could be worse if we used a broadcast approach.

After identifying the users inside the danger zones, the next objective is to identify the best way to reach these people. Our system processes contextual elements (connectivity) to pick the appropriate channel and media format for each user for each situation based on our contextual model. The channel and media format are described in the system output file as well.

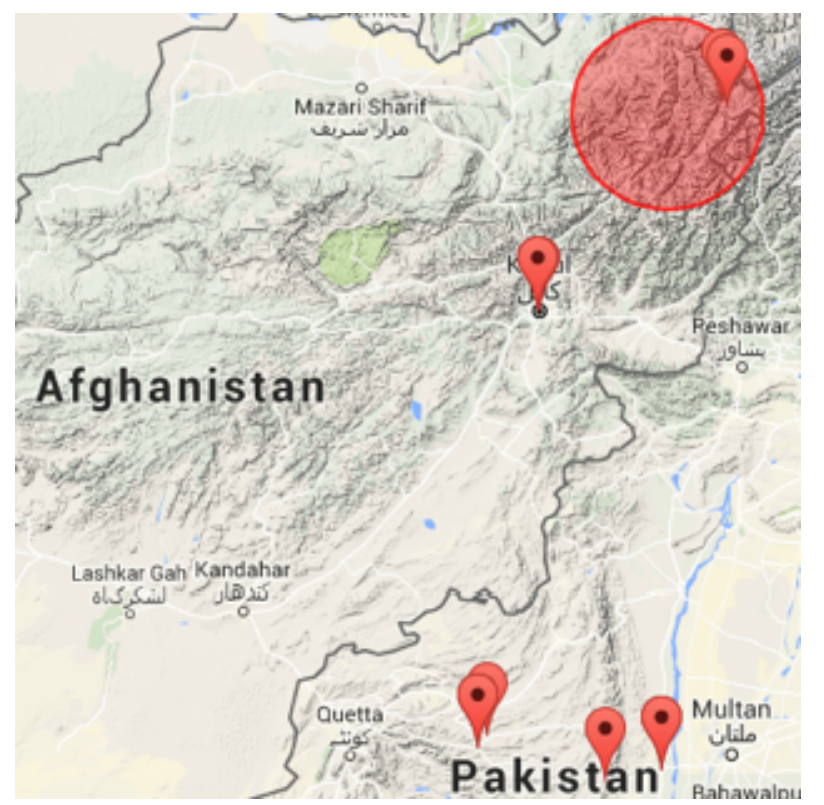

Figure 4: Representation of potential victims selection. The circle represents CAP polygon danger zone. Markers represent people. Only people within danger zone is contacted.

\section{CONCLUSION}

This work presents a conceptual architecture, context model, and a system prototype to support message dissemination in context-aware crisis communication systems. The proposed solution focuses on identifying potential victims to reduce the number of notifications of users who are not in immediate danger, besides defining a strategy to contact the users over different channels and media formats.

The main contributions of this work are the proposed system prototype, the contextual model using Petri Net, and the conceptual architecture which can be used to design other context sensitive crisis communication systems.

For evaluation, we use real emergency data from GDACS and INMET databases. The process shows that strategy used to identify potential victims seems to be more effective than others. Only users in danger zones are selected to be contacted and the way to establishing this communication is based on contextual data from the emergency and the users. As future work we can list: (i) a real distribution module implementation; (ii) evaluation of multiple media formats; and (iii) perform more case studies with larger datasets.

\section{ACKNOWLEDGMENTS}

The authors would like to thank the Brazilian National Institute of Meteorology (INMET) for providing the emergency data used in the evaluation process.

This research was funded by Foundation for Research Support of the State of Bahia (FAPESB).

\section{REFERENCES}

[1] Z. Aarab, R. Saidi, and M. D. Rahmani. Context modeling and metamodeling: A state of the art. In Proceedings of the Mediterranean Conference on 
Information $\&$ Communication Technologies 2015, pages 287-295. Springer, 2016.

[2] S. Banerjee, D. Mukherjee, and P. Misra. 'what affects me?': A smart public alert system based on stream reasoning. In Proceedings of the 7th International Conference on Ubiquitous Information Management and Communication, ICUIMC '13, pages 22:1-22:10, New York, NY, USA, 2013. ACM.

[3] R. Barros, P. Kislansky, L. Salvador, R. Almeida, M. Breyer, and L. Gasparin. Edxl-rescuer ontology: Conceptual model for semantic integration. In Proceedings of the 12th International ISCRAM Conference, 2015.

[4] A. Dey, G. Abowd, and D. Salber. A conceptual framework and a toolkit for supporting the rapid prototyping of context-aware applications. Human-Computer Interaction, 16(2):97-166, 2001.

[5] DHA. Internationally Agreed Glossary of Basic Terms Related to Disaster Management. United Nations Department of Humanitarian Affairs., 1993.

[6] GDACS. Global disaster alerts coordination system. www.gdacs.org, 2004. Accessed in 04-01-2017.

[7] IBGE. Perfil dos municipios brasileiros: Gestão de riscos e resposta a desastres. http://goo.gl/WC4dzC, 2014. Accessed in 04-01-2017.

[8] N. Jing, Y. Li, and Z. Wang. A context-aware disaster response system using mobile software technologies and collaborative filtering approach. In Computer Supported Cooperative Work in Design (CSCWD), Proceedings of the 2014 IEEE 18th International Conference on, pages 516-522. IEEE, 2014.

[9] M. Klafft and H. G. Ziegler. A concept and prototype for the integration of multi-channel disaster alert systems. In Proceedings of the 7th Euro American Conference on Telematics and Information Systems, page 20. ACM, 2014.

[10] A. Malizia, P. Acuña, T. Onorati, P. Díaz, and I. Aedo. Cap-ones: an emergency notification system for all. International Journal of Emergency Management, 6(3-4):302-316, 2009.

[11] T. Murata. Petri nets: Properties, analysis and applications. Proceedings of the IEEE, 77(4):541-580, 1989.

[12] B. Newsome. A Practical Introduction to Security and Risk Management. SAGE Publications, 2013.

[13] OASIS. Common alerting protocol version 1.2. https://goo.gl/g1OJJG, 2010. Accessed in 04-01-2017.

[14] Scotland and S. S. Executive. The Civil Contingencies Act 2004 (Contingency Planning) (Scotland) Regulations 2005. Scottish Statutory Instruments Series. Stationery Office, 2005.

[15] T. Strang and C. Linnhoff-Popien. A context modeling survey. In Workshop Proceedings, 2004.

[16] T. L. Tinker and E. Vaughan. Risk and crisis communications: Best practices for government agencies and non-profit organizations. Booz, Allen, Hamilton, 2010.

[17] UNEP. Pell and floods a community-based approach for disaster reduction. http://goo.gl/k32pLM, 2004. Accessed in 04-01-2017.

[18] V. Vieira, P. Tedesco, and A. C. Salgado. Designing context-sensitive systems: An integrated approach. Expert Systems with Applications, 38(2):1119-1138, 2011. 